\title{
Kinetics of Aggregation in Charged Nanoparticle Solutions Driven by Different Mechanisms
}

\author{
S. Abbas ${ }^{1 *}$, I. Yadav ${ }^{1}$, Sugam Kumar ${ }^{1}$, V. K. Aswal ${ }^{1}$ and J. Kohlbrecher ${ }^{2}$ \\ ${ }^{1}$ Solid State Physics Division, Bhabha Atomic Research Centre, Mumbai 400 085, India \\ ${ }^{2}$ Laboratory for Neutron Scattering, Paul Scherrer Institut, CH-5232 PSI Villigen, Switzerland \\ "abbas@barc.gov.in
}

\begin{abstract}
The structure and kinetics during aggregation of anionic silica nanoparticles as induced through different mechanisms have been studied by dynamic light scattering (DLS) and small-angle neutron scattering (SANS). Three different additives, namely an electrolyte $(\mathrm{NaCl})$, cationic protein (lysozyme) and non-ionic surfactant (C12E10) were used to initiate nanoparticle aggregation. Electrolyte induced aggregation can be explained by DLVO interaction, whereas depletion interaction (non-DLVO interaction) is found responsible for nanoparticle aggregation in case of nonionic surfactant. Unlike these two cases, strong electrostatic attraction between nanoparticle and oppositely charged protein results into protein-mediated nanoparticle aggregation. The electrolyte induced aggregation show quite slow aggregation rate whereas protein mediated as well as surfactant induced aggregation takes place almost instantaneously. The significant differences observed in the kinetics are explained based on range of interactions responsible for the aggregation. In spite of differences in mechanism and kinetics, the nanoparticle clusters are found to have similar fractal morphology (fractal dimension $\sim 2.5$ ) in all the three cases.
\end{abstract}

Keywords: Silica nanoparticle, fractal aggregate, colloids, SANS.

PACS: 81.07.-b, 61.05.fg, 87.15.nr, 78.35.+c

\section{INTRODUCTION}

The colloidal stability is a force-balanced process between two classes of forces: attractive and repulsive where the dominance of repulsive forces preserves the stability of colloidal system while the dominance of attractive forces leads to the spontaneous selfassembly and thus aggregation of the colloidal system [1]. The intrinsic and proper balance of attractive and repulsive interparticle forces gives rise to the formation of desired sized particle clusters having unique characteristics and usefulness in multitude applications ranging from molecular biology to food industry $[2,3]$.

In general, the colloidal aggregation requires the physical processes to bring particles in contact with each other for thermodynamic stability. This can be attained by modifications in interparticle interactions through introduction of selective additives or by changing solution conditions. For example, the presence of electrolyte screens the electrostatic repulsion between the charge stabilized particles resulting into particle aggregation whereas addition of oppositely charged macromolecule causes macromolecule mediated particle aggregation [4, 5]. The morphology, size and the kinetics of aggregation depends on the properties of the particles, solution conditions as well as characteristics of additives, if any.

In the present work, we have studied the kinetics of charged silica nanoparticle aggregation induced by adding an electrolyte $(\mathrm{NaCl})$, cationic protein (Lysozyme) and non-ionic surfactant (C12E10). Dynamic light scattering (DLS) and small-angle neutron scattering (SANS) have been used to characterize these systems. DLS is used mainly for kinetics of the aggregation whereas SANS is utilized for determining the structure of the aggregates.

\section{EXPERIMENTAL}

Electrostatically stabilized colloidal suspensions of silica nanoparticles (Ludox LS30) and additives $[\mathrm{NaCl}$, protein Lysozyme and surfactant decaoxyethylene n-dodecylether (C12E10)] were purchased from Sigma-Aldrich. Samples were prepared by dissolving $1 \mathrm{wt} \%$ amount of silica nanoparticles and varying amount of additives in mixed $\mathrm{H}_{2} \mathrm{O}$ for DLS and in $\mathrm{H} 2 \mathrm{O} / \mathrm{D}_{2} \mathrm{O}$ mixed solvent 
for SANS as per contrast requirement. DLS measurements were carried out on nanoparticle size analyzer SZ-100 (Horiba, Japan). Small-angle neutron scattering experiments were performed at SANS-I facility, Paul Scherrer Institut, Switzerland. Corrections were made for background and empty cell contributions and data were normalized to absolute cross-sectional unit using standard procedures.

\section{DATA ANALYSIS}

In DLS, the intensity autocorrelation function $\left[\mathrm{g}^{2}(\tau)\right]$ of scattered light as a function of delay time $(\tau)$ is measured. This correlation function is related to the effective hydrodynamic size $\left(2 \mathrm{R}_{\mathrm{H}}\right)$ of the particles and can be given by [6]

$$
g^{2}(\tau)=A \exp \left(-2 \frac{k T}{6 \pi \eta R_{H}} Q^{2} \tau\right)+B
$$

where A represents a factor related to instrument parameters and $\mathrm{B}$ denotes the base line. $\mathrm{k}$ is the Boltzmann's constant, $\eta$ is the solvent viscosity, and T is the absolute temperature.

In SANS, one measures the coherent scattering intensity as a function of wave vector transfer [Q $=(4 \pi \sin \theta) / \lambda ; 2 \theta$ is scattering angle, $\lambda$ is wave length of neutrons] which can be given by [7]

$$
I(Q)=n V^{2}\left(\rho_{p}-\rho_{s}\right)^{2} P(Q) S(Q)+B K G
$$

where $n$ is the number density of particles and $V$ is particle volume. $\rho_{p}$ and $\rho_{\mathrm{s}}$ are scattering length densities of particle and solvent, respectively. $P(Q)$ is form factor and $S(Q)$ is interparticle structure factor. $B K G$ is a constant representing incoherent background. The expressions of $\mathrm{P}(\mathrm{Q})$ for different shapes and $\mathrm{S}(\mathrm{Q})$ for different interactions are given in literature [7].

\section{RESULTS AND DISCUSSION}

Fig. 1 shows the SANS data of $1 \mathrm{wt} \%$ silica nanoparticles with different amount of three additives ( $\mathrm{NaCl}$, lysozyme and $\mathrm{C} 12 \mathrm{E} 10$ ) in $\mathrm{H}_{2} \mathrm{O} / \mathrm{D}_{2} \mathrm{O}$ mixed solvent for which micelles and proteins are contrastmatched. The inset shows the SANS data of $1 \mathrm{wt} \%$ of the pure components (silica, lysozyme and C12E10). The structural parameters of the pure components are given in table 1 . The data for the mixed systems have been collected at the additive concentrations where all the nanoparticles are aggregated. It can be seen that the significantly different amounts of additives are required to provoke nanoparticle aggregation due to completely different interactions involved. In case of electrolyte, it screens the electrostatic repulsion between the charged nanoparticles which results into a net attraction leading to particle aggregation (DLVO). The presence of oppositely charged protein molecules

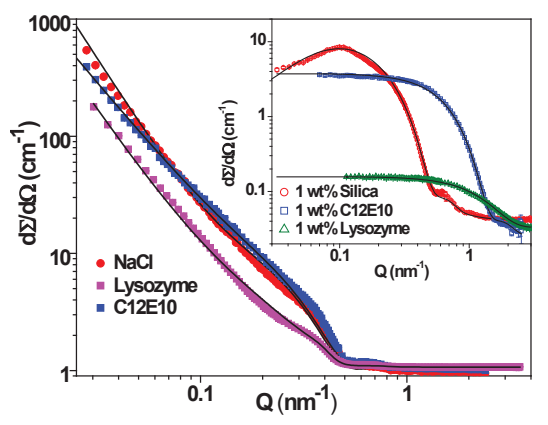

FIGURE 1. SANS data of $1 \mathrm{wt} \%$ Silica nanoparticles mixed with $\mathrm{NaCl}(2.0 \mathrm{M})$, lysozyme $(0.05 \mathrm{wt} \%)$ and $\mathrm{C} 12 \mathrm{E} 10$ $(0.1 \mathrm{wt} \%)$. A small amount $(10 \mathrm{mM})$ of salt has been added with $\mathrm{C} 12 \mathrm{E} 10$ in order to make electrostatic repulsion to be comparable to depletion attraction so that particle clustering can be observed.

mediates the nanoparticle aggregation. On the other hand, non-ionic micelles cause depletion attraction induced nanoparticle aggregation. The details of these mechanisms have been described in our earlier works [4, 5, 8]. In spite of the completely different mechanisms, the structures of the nanoparticle aggregates are found to be quite similar. The SANS data shows linearity in the low Q region for all three cases, suggesting the fractal nature of the aggregates. No low Q cut off is seen in the data within Q range of our measurements, therefore a value of more than $2 \pi / \mathrm{Q}_{\min }$ for maximum of fractal microstructure length has been used during the analysis. The nanoparticle aggregates are found to have mass fractal nature with almost same fractal dimension (2.5).

TABLE 1. Structural parameters of pure components

\begin{tabular}{lccc}
\hline $\begin{array}{c}\text { System } \\
\text { (1 wt\%) }\end{array}$ & $\begin{array}{c}\text { Semi-major } \\
\text { Axis (nm) }\end{array}$ & $\begin{array}{c}\text { Semi-minor } \\
\text { Axis (nm) }\end{array}$ & $\begin{array}{c}\text { Charge } \\
\text { (e.u.) }\end{array}$ \\
\hline Silica & 7.8 & 7.8 & -40 \\
Lysozyme & 2.4 & 1.4 & +7 \\
C12E10 & 1.8 & 1.8 & - \\
\hline
\end{tabular}

Though the structure of nanoparticle aggregates formed through three different ways are similar, the kinetics of their formation is found to be significantly different. Fig. 2 shows the variation of effective aggregate size with time as measured by DLS. It is observed that the nanoparticle aggregation driven by lysozyme and $\mathrm{C} 12 \mathrm{E} 10$ is quite fast in the beginning and soon (within a few minutes) attains a saturation value. On the other hand, a very slow kinetics is involved in the case of electrolyte induced aggregation. The size of the aggregates increases slowly with time and proceeds towards same saturation value as observed in other two cases after about 24 hrs. Moreover, relatively quite low concentration of protein $(0.05 \mathrm{wt} \%)$ and surfactant $(0.1$ $w t \%)$ is required to induce the aggregation whereas quite higher amount (1-2 M) of $\mathrm{NaCl}$ is needed for the same. The differences observed in the kinetics and the 
amounts of the additives are attributed to the nature of interactions responsible for the aggregation. In the cases of protein and surfactant, the responsible interactions (electrostatic and depletion, respectively) are relatively long range compared to van der Waals interaction which is the driving force for electrolyte induced aggregation. Long range attractions are able to bring nanoparticles immediately in contact whereas aggregation controlled by short range forces depends on particle diffusion, exhibiting slow kinetics.

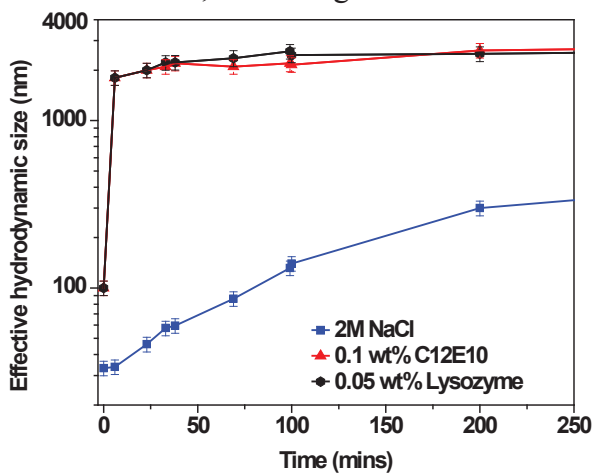

FIGURE 2. Variation of effective aggregate size in $1 \mathrm{wt} \%$ silica nanoparticles mixed with three additives with time as measured by DLS.

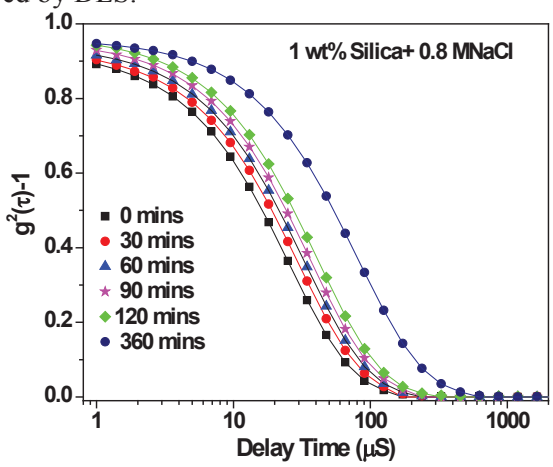

FIGURE 3. DLS auto correlation functions of $1 \mathrm{wt} \%$ silica with $0.8 \mathrm{M} \mathrm{NaCl}$.

The kinetic behavior of electrolyte induced aggregation of nanoparticles is studied in the $\mathrm{NaCl}$ concentration range of 0 to $2 \mathrm{M}$. The evolution of aggregate size for a representative salt concentration $(0.8 \mathrm{M})$ at the onset of aggregation is depicted in Fig. 3 where the DLS autocorrelation functions measured at different times are plotted. One can see the growth in the effective size as reflected in the broadening of the autocorrelation functions. The Variation of effective aggregate size for different salt concentrations with time is shown in Fig.4. The growth of the particle aggregate is quite slow and varies linearly with time in the beginning. Once the formation of aggregates sets in (salt conc. $\sim 1.2 \mathrm{M}$ ), aggregation rate follows power law $\left(\mathrm{a}+\mathrm{bx} \mathrm{x}^{\mathrm{c}}\right.$, c being the growth rate) with growth rate increasing monotonically with salt concentration.

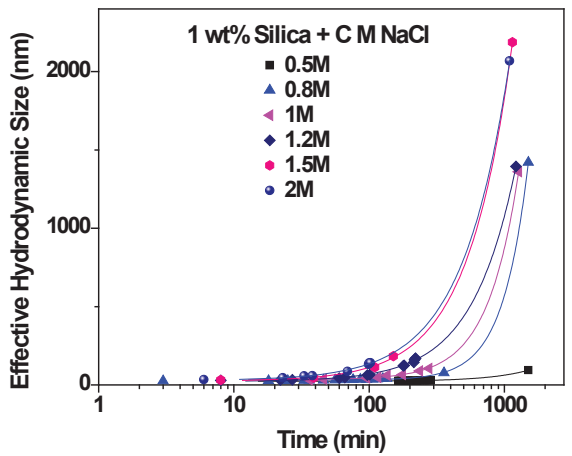

FIGURE 4. Variation of effective aggregate size in $1 \mathrm{wt} \%$ silica nanoparticles in presence of different salt concentrations with time as measured by DLS. The fitted lines show power law behavior of the aggregation.

\section{CONCLUSIONS}

The structure and kinetics of aggregation in anionic silica nanoparticles as induced through different interactions have been studied using SANS and DLS. The nanoparticle aggregation is driven by addition of an electrolyte $(\mathrm{NaCl})$, cationic protein (lysozyme) and non-ionic surfactant (C12E10). The nanoparticle aggregates formed are found to have mass fractal nature having a fractal dimension $(\sim 2.5)$ in all three cases. Albeit the employment of different kind of interactions, the formation of similar kind of final aggregates suggest a universal behavior of such structures. Interestingly, the completely different kinetics behavior is observed for the three cases. While the aggregation in case of lysozyme and C12E10 is quite fast, the kinetics involved in salt induced aggregation is found to be comparatively slow. The differences observed in the kinetics can be understood in terms of nature of interactions involved.

\section{REFERENCES}

1. Y. Liang, N. Hilal, P. Langston and V. Starov, Adv. Colloid Interface Sci. 134-135, 151 (2007).

2. R. Mezzenga, P. Schurtenberger, A. Burbidge and M. Michel, Nature Materials 4, 729-740 (2005).

3. A.E. Nel, L. Mädler, D. Velegol, T. Xia, E.M.V. Hoek, P.Somasundaran, F. Klaessig, V. Castranova and M. Thompson, Nat. Mater. 8, 543-557 (2009).

4. S. Kumar, V. K. Aswal and P. Callow, Langmuir 30, 1588-1598 (2014).

5. A.J. Chinchalikar, V.K. Aswal, J. Kohlbrecher and A.G. Wagh Chem. Phys. Lett. 542, 74-80 (2012).

6. P. A. Hassan, S. Rana, and G. Verma Langmuir 31, 3-12 (2015).

7. J. S. Pedersen, Adv. Coll. Inter. Sci. 70, 171-210 (1997).

8. D. Ray and V. K. Aswal J. Phys.: Condens. Matter. 26, $035102(2014)$ 